\title{
LE CUEILLEUR DE MÉTAPHORES Analyser des situations de communication grâce aux métaphores selon George Lakoff
}

\section{Gérard Pirotton ${ }^{1}$}

Relativement peu connu dans le champ des sciences de la communication, George Lakoff ${ }^{2}$ fait pourtant des propositions qui ne peuvent laisser indifférent dès lors que l'on se préoccupe de comprendre avec finesse des situations de communication. C'est de cette pertinence des thèses de cet auteur qu'entend rendre compte cet article, qui s'appuie sur les résultats d'une recherche doctorale centrée sur l'analyse d'interactions en situation d'apprentissage.

Peut-on communiquer (avec) des images, et avec des mots ? Délibérément imprécise dans sa formulation, cette interrogation offre toutefois une première approximation de ce dont va traiter cet article, ce qu'il s'agit alors d'affiner, en abordant successivement trois questions. Dans quel sens va-t-on entendre ici le terme "image" ? On aura recours pour cela aux travaux de George Lakoff dont les principaux apports seront l'objet de la première section. Ainsi définies, comment identifier de telles structures imagées dans les productions

1 Docteur en sciences sociales (information et communication).

2 Son seul ouvrage publié à ce jour en langue française: G. LAKOFF, M. JOHNSON, Les métaphores dans la vie quotidienne, Paris, Éd. de Minuit, 1985 (1980, pour l'édition originale). 
verbales recueillies en situations naturelles? Cette dimension méthodologique constituera la partie centrale de cet article. Troisièmement, qu'est-ce que de tels outils méthodologiques peuvent apporter à la compréhension de ce qui se joue dans des situations d'interaction? L'article présente succinctement les résultats d'une recherche portant sur l'analyse de situations de formation des adultes. Enfin, on conclura par quelques éléments de réflexion de portée générale sur la méthode proposée.

\section{Ces métaphores qui nous font vivre}

Dans le concert contemporain des théories reconnues, Lakoff présente des conceptions quelque peu à la marge de celles auxquelles on fait généralement place dans les grandes revues. Il soutient que le procédé métaphorique est le mécanisme fondamental de l'entendement humain, un procédé par lequel nous pouvons :

Comprendre quelque chose (et en faire l'expérience) en termes de quelque chose d'autre ${ }^{1}$.

Si la projection métaphorique caractérise la manière dont les êtres humains attribuent du sens aux mondes qui les entourent et cherchent à s'y orienter, cette caractéristique est particulièrement observable au niveau du langage. Ce mécanisme général de compréhension, cette projection du connu sur l'inconnu, ne décrit pas la seule activité cognitive individuelle. Les structures schématiques et imagées qui servent de support à cette projection sont culturelles : elles sont le fait d'une communauté linguistique et ne ressortissent pas de la seule créativité individuelle, privée et éphémère de chaque locuteur en situation. Ce souci d'articulation entre les niveaux individuel et collectif est une des originalités des thèses de Lakoff, c'est aussi ce qui fait l'intérêt d'une confrontation entre ses apports et les questions que l'on travaille en sciences de la communication, dans la mesure où il offre une piste d'articulation entre interaction et cognition, les deux principales négligences du "modèle du code".

1 "Idem, p. 15. 
L'auteur utilise le terme de métaphorel dans un sens assez différent de l'acception philologique du terme. Selon la conception assez particulière de Lakoff, une métaphore est une "structure schématique et imagée" qui se situe, pourrait-on dire en utilisant précisément une métaphore spatiale, "au-dessous" de la formulation de surface observable dans les discours. Dans cette conception, les mots sont, au mieux, des traces indicatrices des métaphores, que l'analyse permet de dégager. De plus, une même métaphore sousjacente peut donner lieu à une série de formulations de surface différentes, leur origine commune n'apparaissant qu'à l'analyse.

Dans cette approche, il n'y a pas ici de distinction entre un sens propre, qui serait non figuratif et un sens imagé, qui aurait quant à lui recours à la métaphore. On ne peut échapper à l'usage des métaphores, elles nous sont indispensables pour donner sens aux univers qui nous entourent. La signification ne s'interprète pas comme la correspondance d'un signe à un objet de cet univers mais comme la projection d'un domaine d'expérience connu à une portion de ces univers au sein desquels nous cherchons à nous orienter. Dans cette conception, la métaphore désigne tout à la fois une structure schématique et imagée, une structure de connaissance, une logique de raisonnement propre à un domaine d'expérience, et le procédé de projection quasi cartographique (mapping) par lequel cette structure est utilisée pour donner sens à un autre domaine et nous y orienter.

Prenons un exemple simple : "Jean opère sa voiture". Le procédé métaphorique ne consiste pas en l'usage d'un terme emprunté à la médecine pour le transposer dans le domaine de la mécanique. Il s'agit davantage d'une formulation de surface indicatrice d'une métaphore sous-jacente, caractérisant notre culture et que l'on pourrait formuler ainsi : "UNE-VOITURE-EST-UNE-PERSONNE". Un locuteur aura recours à cette structure, en disant par exemple que "sa voiture est morte", que "le moteur est grippé", qu'"elle bouffe de l'huile", qu" "elle lui a encore fait des siennes" ou qu' "elle a rendu l'âme".

On voit bien ainsi que le savoir relatif au domaine des personnes et la structure de connaissance qui y correspond sont projetés sur celui de l'automobile, en établissant des correspondances, point à point,

1 Pour une moins brève présentation des thèses de l'auteur, on pourra lire : G. PIRotTon, "Métaphore et communication pédagogique, Vers un usage délibéré de la métaphore à des fins pédagogiques", Recherches en communication, $\mathrm{n}^{\circ} 2$, 1994, pp. 73-88. 
entre ces deux domaines et autorisant ainsi chacune des formulations de surface citées ci-dessus, comme autant de mises en œuvre de cette métaphore.

Cette dernière est partagée par les différents locuteurs d'une même langue. Cette dimension culturelle est une des composantes des propositions de Lakoff, soulignant ainsi le fait que la métaphore ne concerne pas le seul niveau cognitif individuel mais relève aussi du niveau culturel. On concédera volontiers le caractère schématique de cette présentation. Toutefois, elle peut sans doute suffire ici pour notre propos.

\section{Identifier des métaphores dans des situations naturelles}

Il n'est pas aisé d'extraire des travaux de Lakoff une méthodologie opérationnelle d'identification des métaphores, dans le sens qu'il attribue à ce terme. De plus, l'auteur a essentiellement fondé ses propres recherches sur l'analyse des expressions idiomatiques issues de la vie quotidienne, tandis que les situations qui vont être citées sont des interactions en situation naturelle. En l'occurrence, il s'agit plus précisément de l'observation de séances de cours dans une formation pour adultes centrée sur l'apprentissage de l'approche systémique, rapportée à l'intervention sociale. Les pratiques pédagogiques qui y ont cours tablent sur les interactions entre participants. Cette recherche a donc nécessité l'élaboration d'outils méthodologiques spécifiques. Leur présentation constitue l'objet de la présente section.

On va tout d'abord rendre compte des caractéristiques des matériaux pour lesquels la méthodologie en question a été élaborée. On présentera ensuite les différentes techniques destinées à préparer le matériau brut, les outils de diagnostic préparatoires aux analyses et enfin le modus operandi d'identification des métaphores. A la fin de l'article, on discutera cet ensemble d'un point de vue plus général, notamment quant aux limites de la pertinence de cette approche, quant à l'articulation des outils d'analyse et des questions de recherche et quant à la position du chercheur à l'égard de ceux que l'on a coutume d'appeler les sujets. 


\subsection{Les matériaux appropriés}

Les matériaux bruts qui se prêtent le mieux à un diagnostic des métaphores sont des retranscriptions de productions verbales récoltées en situation naturelle. Dès lors, en amont de cette retranscription, se posent une série de questions et notamment le rapport observateur-

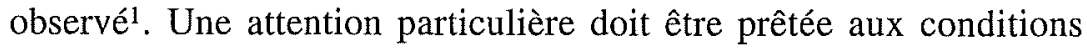
dans lesquelles ces données brutes vont pouvoir être récoltées. Il appartient au chercheur de veiller à ce que les conditions de collecte des données permettent une expression aussi spontanée que possible des personnes dont on veut ensuite explorer les discours. Le chercheur se doit de faire preuve d'un savoir-faire relationnel autant que de vigilance pour s'assurer que ces conditions soient remplies ${ }^{2}$.

La retranscription dactylographiée des enregistrements ne demande pas moins de soin : l'identification des voix et l'exactitude scrupuleuse des termes utilisés, par exemple, demandent vigilance et précision. Il est ainsi plus correct de mentionner le caractère inaudible de certains passages plutôt que de chercher à être complet en "devinant" les termes manquants.

\subsection{Illustration}

Dans les pages qui vont suivre, nous allons reprendre à plusieurs reprises une même extrait, comme illustration de l'usage qui peut être fait des outils méthodologiques que nous avons élaborés ${ }^{3}$. Ce premier extrait demande à être quelque peu présenté. Il apparaît en effet dans

1 Voir notamment: M. GRAwitz, Méthodes des Sciences Sociales, Paris, Dalloz, Précis, (9édition), 1993. Voir spécifiquement le $\S 2$ de la Section 1 du Chapitre 1 du Titre 2 (pp. 582 et sv.) ainsi que le $\S 3$ de la Section 1 du Chapitre 2 de ce même Titre 2 (pp. 686 et sv.). On reprendra cette question à la fin de l'article.

2 Voir : L. Albarello, F. DignefFe, J.-P. HiernauX, C. Maroy, D. RuQuoy, P. DE SAINT-GEORGES, Pratiques et méthodes de recherche en sciences sociales, Paris, Armand Colin, Cursus, 1995. Voir particulièrement: D. RuQuoY, "Situation d'entretien et stratégie de l'interviewer", pp. 59-82 et C. MAROY, "L'analyse qualitative d'entretien", pp. 83-110.

3 Si cette option présente des avantages -il n'est donc pas nécessaire de "rentrer" chaque fois dans la richesse d'un nouvel exemple-elle présente aussi l'inconvénient d'une restriction excessive, attachée aux caractéristiques particulières du fragment en question, au contexte dans lesquels ces matériaux ont été récoltés et à l'analyse que nous en avons faite. Nous prenons alors le parti de mettre en exergue ces spécificités à ce stade de l'exposé, puis de dégager ultérieurement des considérations plus générales. 
des circonstances précises, construites en fonction des préoccupations de la recherche.

Le travail en sous-groupes est très régulièrement utilisé en formation des adultes. La consigne qui est alors fournie aux participants consiste en l'élaboration commune d'une tâche qu'un porteparole aura ensuite à présenter à l'ensemble du groupe. Dans les observations qui ont précédé celle-ci, on a dû constater le poids restrictif que fait peser sur la diversité des métaphores utilisées lors des échanges entre étudiants une consigne de travail qui les invite à procéder à une analyse commune d'une situation complexe. Sur base de ce constat, on a donc proposé à un sous-groupe d'étudiants de travailler avec une consigne particulière. On leur a demandé, non de se mettre d'accord sur une analyse commune ${ }^{1}$, mais tout au contraire d'expliciter et d'examiner les différences de conceptions qui n'allaient pas manquer d'apparaître entre leurs avis respectifs. Objet de leur discussion : leurs conceptions de la communication.

Dans les pages qui vont suivre, à l'occasion des différentes reprises du même exemple, on rappellera au besoin ce contexte particulier afin d'éviter d'éventuelles mésinterprétations. Notons encore que, dans la typographie, les mots soulignés sont des mots accentués par l'intonation des locuteurs.

\subsection{La préparation des matériaux}

Un des problèmes à affronter est celui de la maniabilité du matériau. Lorsque la retranscription dactylographiée s'étale sur plus de deux ou trois pages, il devient difficile de consacrer une attention égale et de conserver en mémoire d'égale manière l'ensemble des détails du matériau. Plus il sera volumineux, plus il sera nécessaire d'avoir recours à des repères précis, de manière à faire référence à des passages déterminés ${ }^{2}$. Outre la numérotation des pages, on peut aussi numéroter chacune des lignes de la dactylographie intégrale.

1 Lors des analyses qui ont précédé la mise en place d'un tel dispositif, on avait constaté que la consigne d'une production commune se conjuguait avec la disparition des métaphores plus marginales. Ne sont généralement retenues pour la phase de mise en commun que celles qui sont susceptibles de permettre un accord entre les protagonistes... et recueillir l'assentiment de l'enseignante !

2 Quoiqu'il aborde cette question à propos d'une autre grille d'analyse, Jean-Pierre Hiernaux pose cette même question et suggère des instruments pour l'affronter qui peuvent aussi s'avérer une source de réflexion. Voir : J.-P. HIERnauX, "Analyse 
Anémone : (à Claude) Mais tu disais que, tu parlais des acquis que l'on avait dans la vie, et que de recevoir, la notion de recevoir et de donner, mais... moi, je n'imagine pas la communication comme ça. La communication, elle est là, omniprésente, quoi, partout.

Claude : Plus spontanée, tu veux dire ? Mais c'est ce que j'ai dit tantôt, quoi, enfin. (Denise entre dans le local. Grincement de porte. Quelques mots inaudibles), c'est ce que j'ai dit tantôt, on n'a pas besoin de recevoir pour qu'il y ait, donc la communication peut être de manière spontanée, de la personne, quoi. Il n'y a peut-être pas besoin non plus d'acquis.

Anémone : Mais non! Moi, je ne crois pas non plus! C'est quelque chose qui est.

Claude : Inné ?

(...)

Anémone : Qui est.

(...)

Anémone: Mais qu'on ne doit pas nécessairement apprendre à communiquer, qu'on le fait. On peut améliorer sa communication. Mais la communication est. Mais par contre, quand tu parles d'acquis et de vécu, la façon dont on va communiquer est colorée par le vécu qu'on a, les acquis qu'on a, de même que on va comprendre la communication on va la réceptionner, de manière différente, selon le vécu ou les acquis qu'on a. Ça, je crois aussi.

(...)

Claude : Et au départ, qu'est-ce qu'y a alors, quand tu présentes les choses comme ça ?

Anémone: Mais, un individu est, est quelque chose de vivant, un système ouvert, donc il y a communication vers l'extérieur, de toute façon, donc, tu vois?

Claude : Ah oui,

Anémone : Ou sinon, c'est quelque chose de mort, sans communication vers l'extérieur.

Dans les cas de transcription d'un échange où interviennent plusieurs personnes, on peut aussi numéroter chacune des interventions des différents protagonistes. Lorsque la retranscription concerne le discours d'une seule personne, on peut aussi numéroter chacun des paragraphes, tout en sachant que ce découpage en paragraphes ressortit de l'interprétation qui a immanquablement lieu au moment de la retranscription, comme c'est également le cas pour la ponctuation, par exemple.

structurale de contenus et modèles culturels. Application à des matériaux volumineux, in L. AlBARELlo et al., op. cit., pp. $111-144$. 
Anémone : (15) (à Claude) Mais tu disais que, tu parlais des acquis que l'on avait dans la vie, et que de recevoir, la notion de recevoir et de donner, mais,... moi, je n'imagine pas la communication comme ça. La communication, elle est là, omniprésente, quoi, partout.

Claude : (16) Plus spontanée, tu veux dire ? Mais c'est ce que j'ai dit tantôt, quoi, enfin. (Denise entre dans le local. Grincement de porte. Quelques mots inaudibles) c'est ce que j'ai dit tantôt, on n'a pas besoin de recevoir pour qu'il y ait, donc la communication peut être de manière spontanée, de la personne, quoi. Il n'y a peut-être pas besoin non plus d'acquis.

Anémone : (17) Mais non! Moi, je ne crois pas non plus! C'est quelque chose qui est.

Claude : Inné ?

(...)

Anémone : (17) Qui est.

(...)

Anémone : (17) Mais qu'on ne doit pas nécessairement apprendre à communiquer, qu' on le fait. On peut améliorer sa communication. Mais la communication est. Mais par contre, quand tu parles d'acquis et de vécu, la façon dont on va communiquer est colorée par le vécu qu'on a, les acquis qu'on a, de même que on va comprendre la communication on va la réceptionner, de manière différente, selon le vécu ou les acquis qu'on a. Ça, je crois aussi.

(...)

Claude : (18) Et au départ, qu'est-ce qu'y a alors, quand tu présentes les choses comme ça ?

Anémone : (19) Mais, un individu est, est quelque chose de vivant, un système ouvert, donc il y a communication vers l'extérieur, de toute façon, donc, tu vois?

Claude : Ah oui,

Anémone : (19) Ou sinon, c'est quelque chose de mort, sans communication vers l'extérieur.

On peut encore avoir recours à une technique que nous appelons le "bout-à-bout".

Ce procédé consiste à faire figurer les unes à la suite des autres les différentes interventions d'une même personne dans l'ordre où elles sont apparues et à agir de la même façon pour les autres intervenants. Cet outil permet de centrer l'attention du chercheur sur les contributions de chaque interlocuteur en particulier, des contributions qui apparaissent alors quasi hors contexte. Cet artifice permet aussi au chercheur de prendre distance à l'égard de la dynamique de l'échange et permet de mettre au jour des éléments qui 
peuvent passer inaperçus, à la simple lecture de la retranscription intégrale de l'échange tel qu'il s'est déroulé. Cependant, il est souvent nécessaire, pour saisir le sens d'une intervention, de retourner au contexte de son énonciation. Ce contexte ne se réduit d'ailleurs pas aux autres interventions verbales, puisqu'il y a lieu de tenir compte par exemple de l'accompagnement gestuel, du cadre organisationnel, de l'état et de l'histoire des relations entre les locuteurs, etc. La numérotation des interventions, qui permet d'ordonner la retranscription intégrale de l'échange, doit être conservée au sein du "bout-à-bout", ce qui permet un passage aisé d'une matériau à un autre.

A première vue, ce procédé du "bout-à-bout" peut n'apparaître comme pertinent que si l'on cherche à identifier les métaphores de chaque interlocuteur. Précisons alors deux points. D'une part, on ne peut ainsi concevoir les métaphores en termes de "propriété privée" de chaque interlocuteur, alors que ces métaphores relèvent davantage de la culture, au sens anthropologique du terme, des interlocuteurs engagés dans l'échange. D'autre part, les métaphores que l'on peut ainsi identifier chez chaque interlocuteur peuvent davantage s'analyser comme des contributions, des participations à l'échange, lui-même appréhendé comme un seul discours ${ }^{1}$.

\section{ANÉMONE}

Anémone : (15) (à Claude) Mais tu disais que, tu parlais des acquis que l'on avait dans la vie, et que de recevoir, la notion de recevoir et de donner, mais,... moi, je n'imagine pas la communication comme ça. La communication, elle est là, omniprésente, quoi, partout.

Anémone : (17) Mais non! Moi, je ne crois pas non plus! C'est quelque chose qui est.

Anémone : (17) Qui est.

Anémone : (17) Mais qu'on ne doit pas nécessairement apprendre à communiquer, qu'on le fait. On peut améliorer sa communication. Mais la communication est. Mais par contre, quand tu parles d'acquis et de vécu, la façon dont on va communiquer est colorée par le vécu qu'on a, les acquis qu'on a, de même que on va comprendre la communication on va la réceptionner, de manière différente, selon le vécu ou les acquis qu'on a. Ça, je crois aussi.

1 Voir à ce propos la troisième partie de cet article. 
Anémone: (19) Mais, un individu est, est quelque chose de vivant, un système ouvert, donc il y a communication vers l'extérieur, de toute façon, donc, tu vois ?

Anémone : (19) Ou sinon, c'est quelque chose de mort, sans communication vers l'extérieur.

\section{CLAUDE}

Claude : (16) Plus spontanée, tu veux dire ? Mais c'est ce que j'ai dit tantôt, quoi, enfin. (Denise entre dans le local. Grincement de porte. Quelques mots inaudibles) c'est ce que j'ai dit tantôt, on n'a pas besoin de recevoir pour qu'il y ait, donc la communication peut être de manière spontanée, de la personne, quoi. Il n'y a peut-être pas besoin non plus d'acquis.

Claude : Inné ?

Claude : (18) Et au départ, qu'est-ce qu'y a alors, quand tu présentes les choses comme ça ?

Claude : Ah oui,

Ces différents procédés (numérotation, bout-à-bout) peuvent ne pas suffire à rencontrer totalement la difficulté que représente un matériau volumineux. Il est alors nécessaire de procéder à la construction d'extraits pertinents.

Le matériau en lui-même peut fournir des indications permettant de le découper en sous-unités plus maniables. Ainsi, la lecture attentive de la retranscription d'une discussion au cours de laquelle les interlocuteurs changent de "sujet de conversation", permet sans doute de tracer les limites d'extraits plus maniables, des limites dont la légitimité et les critères de pertinence sont alors issus de la dynamique même de l'échange retranscrit, tel qu'il s'est déroulé. Dans ce cas, c'est donc le matériau lui-même qui fournit les indications autorisant la confection de tels fragments : le partage est réalisé sur base de la logique interne du matériau brut à étudier.

Dans d'autres cas, ce seront les questions de recherche qui fourniront les repères nécessaires au découpage justifié du matériau. Ainsi d'une recherche qui se donne pour but de relever les métaphores à partir desquelles les participants à une conversation appréhendent un objet particulier ${ }^{1}$. On conçoit bien qu'en 1'espèce, seules certaines interventions seront pertinentes en regard d'une telle préoccupation,

1 Par exemple leur voiture, pour reprendre le cas cité au début de ce chapitre, en illustration des thèses de Lakoff. 
parmi l'ensemble des éléments que contient le matériau brut. Dans ce cas, les critères de pertinence pour la composition d'extraits relèvent d'une logique externe au matériau brut étudié.

\subsection{Identification des traces}

Recueillir et préparer le matériau ne fournit pas encore la moindre indication quant aux métaphores susceptibles d'être identifiées. Dans la mesure où elles n'apparaissent pas telles qu'elles directement à la surface de l'expression mais qu'elles y affleurent sous des formes diverses, le chercheur va devoir identifier ces affleurements, à partir desquels, par inférence, il s'efforcera de formuler les structures schématiques imagées, les logiques imagées de raisonnement qui leur ont servi de fondement. Comment procède-ton?

Les extraits pertinents étant délimités, c'est donc à l'identification des traces de métaphores qu'il faut s'atteler. Cette opération nécessite des relectures successives, flottantes autant que vigilantes, destinées à bien s'imprégner du matériau. Au fil de ces relectures vont progressivement émerger des termes particuliers, des fragments de phrases ou des expressions idiomatiques, tenus pour indicatifs des façons de raisonner l'objet dont il est question.

Les termes tenus pour des traces de logiques imagées de raisonnement seront signalés en les marquant dans le texte par un grisél. Les fragments d'interventions ou les expressions idiomatiques relevées comme indicatrices potentielles de logiques de raisonnement sont marquées par des signes conventionnels: "<" et ">".

1 Il est bien sûr toujours possible d'utiliser d'autres marquages, pour autant qu'ils se distinguent sans ambiguitté de tout autre marquage, comme le gras, le souligné ou le double-souligné, qui pourraient par exemple être déjà utilisés pour rendre compte, dans la dactylographie, du caractère accentué de certains termes par le locuteur dont on retranscrit les propos. 
1 Anémone : (15) (à Claude) Mais tu disais que, <tu parlais des aequis que l'on

2 avait dans la vie, et que de reevoir, la notion de reeevoir et de donner; $>$ 3 mais,... moi, je n'imagine pas la communication comme ça. <La

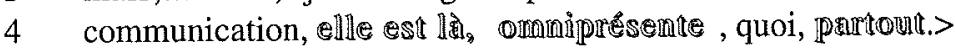

5 Claude : (16) Plus spontanée, tu veux dire ? Mais c'est ce que j'ai dit tantôt, 6 quoi, enfin. (Denise entre dans le local. Grincement de porte. Quelques mots 7 inaudibles) c'est ce que j'ai dit tantôt, on n'a pas besoin de recevoỉr pour 8 qu'il y ait, donc la communication peut être de mainì șe spomtamée, de la

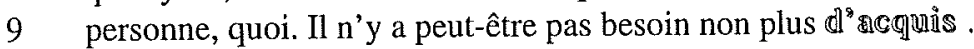

10 Anémone : (17) Mais non! Moi, je ne crois pas non plus! <C'est quelque 11 chose qui est.>

12 Claude : Inné ?

13 (...)

14 Anémone : (17) <Qui est.>

15 (...)

16 Anémone: (17) <Mais qu'on ne doit pas nécessairement apprendre à 17 communiquer, qu'on le fait. $>$ On peut améliorer sa communication. Mais <la 18 communication est.> Mais par contre, quand tu parles d'aequis et de véeu, 19 < la fisçon dont on va communiquer est colorée par le vêEn qu'on a>, les 20 nequaris qu' on de même que on va comprendre la communication on va la

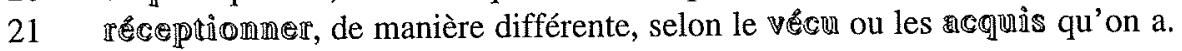
22 Ça, je crois aussi.

23 (...)

24 Claude : (18) Et ân dênpart, qu'est-ce qu'y a alors, quand tu présentes les 25 choses comme ça?

26 Anémone : (19) Mais, un individu est, est quelgue chose de vivant, un 27 système ouvert, donc il y a communication vers l'extérieur, de toute façon, 28 donc, tu vois ?

29 Claude: Ah oui,

30 Anémone: (19) Ou sinon, c'est quelque chose de mort, sans 31 communication vers l'extérieur.

Note : Dans le passage ci-dessus, les mots rayés correspondent à des termes pour lesquels on a estimé qu'ils étaient prononcés, non comme un indice de l'usage d'une métaphore déterminée, mais davantage comme une simple reprise du vocabulaire de l'interlocuteur. C'est ici le lieu de rappeler que, dans le fragment considéré, les interlocuteurs sont engagés dans un échange qui a pour consigne particulière, non de se mettre d'accord sur une conception commune de la communication, mais d'identifier entre eux les différences de conceptions. C'est ce contexte particulier qui a autorisé les juges à procéder de la sorte. Ailleurs, dans d'autres contextes, la 
reprise par les interlocuteurs des métaphores de leurs partenaires pourrait s'interpréter autrement.

Ces termes vont alors être tenus comme des éléments constitutifs mais encore éparts d'une ou plusieurs structures, comme autant d'indices qu'il s'agira ensuite de regrouper et d'articuler entre eux. $\mathrm{Ce}$ qui permet alors d'inférer des structures schématiques et imagées, des logiques de raisonnement à partir desquelles l'objet est abordé par les interlocuteurs, dans cette situation particulière.

Ainsi, dans l'extrait qui nous sert d'illustration, des termes comme "recevoir, donner, acquis, réceptionner, vers,..." peuvent être regroupés et ont été tenus ensemble pour indicatifs de la présence d'une structure schématique et imagée qui se caractérise par l'idée générale de transfert, selon laquelle "communiquer consister à transférer quelque chose". Les autres traces peuvent ainsi être regroupées dans d'autres sous-ensembles, permettant à leur tour de formuler d'autres structures schématiques imagées ${ }^{1}$.

\subsection{Cueilleurs de métaphores}

On peut alors consigner cet ensemble dans un tableau au sein duquel figurent, comme titre des colonnes, les logiques imagées de raisonnement identifiées et comme contenu à ces colonnes, le recrutement des traces qui y correspondent.

Inversement, l'identification d'une logique imagée de raisonnement peut mettre le chercheur sur la piste d'autres traces moins saillantes qui, quoique présentes, n'auraient peut-être pas été identifiées comme telles, sans que cette logique n'ait été préalablement dégagée sur base d'autres traces plus explicites quant à leurs fondements métaphoriques. Cette circularité (des traces permettent d'identifier des logiques; des logiques permettent d'identifier des traces) parait non comme un mal nécessaire inévitable, mais constitue plutôt une caractéristique de ce genre de méthode dont les vertus heuristiques tiennent précisément à ce déploiement réitératif.

1 On notera que selon les propositions de Lakoff, plusieurs métaphores peuvent être présentes au sein d'un même discours, non à titre exceptionnel, comme une tolérance du modèle, mais au contraire comme une de ses caractéristiques. Dans la mesure où chaque métaphore met en lumière et occulte des aspects différents, cela rend nécessaire le recours à des métaphores différentes. A ce titre, elles sont donc davantage complémentaires qu'antagonistes. 
Osons une illustration naturaliste. Entre une métaphore conceptuelle et ses occurrences langagières particulières, il y a un rapport du même type qu'entre un mycélium et des champignons. A l'occasion d'une balade, un promeneur non averti constatera bien la présence de l'un ou l'autre champignon. Par contre, l'amateur saura qu'ils éclosent sur les bords d'une toile souterraine et donc invisible que constitue le mycélium, une toile circulaire. Dès lors, cet amateur sera sans doute capable, sur base de quelques champignons qu'il observe, de repérer l'emplacement du mycélium et donc de recomposer la courbure du cercle le long duquel il a de fortes chances d'en découvrir d'autres.

La fréquentation des écrits de Lakoff ${ }^{1}$ et une bonne connaissance du contexte où ont été réalisées les transcriptions sont des éléments qui facilitent cette identification des traces. De manière générale, la dimension spatiale étant importante dans les thèses de Lakoff, les termes indicatifs de cette dimension peuvent souvent être relevés comme traces, soit de façon isolée, soit au sein d'un fragment plus important qui les contiennent.

Enfin, on peut concevoir l'existence de plusieurs degrés de saillance des métaphores ou plus précisément des traces au travers desquelles elles se donnent à voir ${ }^{2}$. Certaines traces seront plus rapidement identifiées que d'autres. Les traces les plus saillantes permettent alors de faire l'hypothèse de l'existence d'une métaphore, que l'on cherchera à confirmer au travers de nouvelles traces que l'on avait pas encore plus relever jusque là. Il est important de çonserver à l'esprit que chaque trace présumée doit être examinée selon qu'elle permet d'inférer le domaine d'expérience qui est projeté sur l'objet discuté.

\subsection{Un collège de Juges}

Bien sûr, la sensibilité, le savoir-faire et l'expérience du chercheur interviennent également dans cette mise en œuvre, mais il est aussi nécessaire d'avoir recours à des juges. Un même document

1 Des listes de métaphores identifiées par Lakoff et ses équipes, chaque fois illustrées par des expressions de la langue anglaise qui les actualise, sont consultables sur les sites suivants :

http://cogsci.berkeley.edu/targets + http://cogsci.berkeley.edu/sources.

2 La question reste toutefois ouverte de savoir si ces différents degrés renvoient à l'objet d'investigation que sont les métaphores ou concernent plutôt les incompétences du chercheur de métaphores... 
peut ainsi être lu et travaillé par plusieurs personnes, familiarisées à cette approche métaphorique, entraînées à la méthode et confrontant ensuite leurs jugements. L'ensemble des matériaux, bruts et préparés comme ci-dessus, leur est remis. Ils ont alors pour tâche d'identifier les traces de métaphores potentielles, mots ou fragments de phrases, qui leur semblent indicatifs de métaphores. Souvent concordantes, quelquefois divergentes, ces lectures sont ensuite comparées. Cette confrontation des lectures et des analyses conduit à ne retenir que les interprétations pour lesquelles un accord argumenté peut être élaboré. A l'expérience, c'est très majoritairement le cas. Pour nuancer l'analyse, on peut aussi restituer les occasions et la portée des éventuels désaccords entre juges. Il peut s'avérer utile d'y revenir ultérieurement.

\subsection{Comptages, tableaux et précautions}

Jusqu'ici, chaque extrait pertinent est tenu pour un seul discours, même si plusieurs interlocuteurs contribuent à le produire. Cette option peut se trouver fondée aux plans théorique et méthodologique. Au plan théorique, parce que, ainsi que nous l'avons déjà signalé, les propositions de Lakoff conçoivent les métaphores davantage comme caractéristiques d'une culture que propres à des individus singuliers. Au plan méthodologique, dans la mesure où, c'est un fréquent fait d'observation, l'examen des conversations en situation naturelle fait apparaître combien il est quelque fois bien difficile, pour des raisons empiriques, de distinguer les productions respectives de chaque interlocuteur, par exemple lorsqu'une phrase est commencée par l'un, poursuivie par l'autre, voire terminée par un troisième.

Ce recensement des traces indicatrices de logiques imagées de raisonnement étant terminé, on peut procéder à une série de comptages, qui peuvent être consignés sous la forme de tableaux.

Ici encore, cette construction de tableaux va dépendre, non pas tant d'une conformité à une méthode généraliste, mais des questions spécifiques qui motivent la recherche. Dans un cas par exemple, on ne se préoccupera pas du nombre de fois qu'une même métaphore est présente au travers de différentes formes linguistiques, mais on se préoccupera davantage de la seule présence ou absence de cette logique imagée de raisonnement. L'importance proportionnelle respective qu'occupe chaque métaphore par rapport aux autres n'est alors pas considérée comme une donnée relevante par rapport aux 
questions de la recherche. Dans un autre cas encore, on pourra se préoccuper non seulement de faire apparaître le nombre de fois que chaque logique imagée de raisonnement identifiée est utilisée, mais encore on voudra en répartir l'usage entre les différents protagonistes d'une conversation que l'on cherche à étudier sous l'angle métaphorique. Dans ces différents cas, les tableaux qui recensent les données identifiées dans l'étape précédente seront présentées, non pas en appliquant une procédure standard mais sous une forme qui permette ensuite une analyse en cohérence avec les questions de recherche.

Le recensement des traces de logiques imagées de raisonnement donne donc lieu à des agrégations qui peuvent prendre des formes diverses, en fonction des questions de recherche. Lorsque celles-ci nécessitent le recours à des comptages ${ }^{1}$, on présentera ces données, soit en valeur absolue, soit en pourcentage, ce qui permet notamment des comparaisons.

\begin{tabular}{|c|c|c|c|c|c|}
\hline X. $10:$ & \multicolumn{5}{|c|}{$\begin{array}{l}\text { Les traces de logiques de raisonnement utilisés par Claude et Anémone } \\
\text { au fil des interventions, dans la troisième partie de l'échange }\end{array}$} \\
\hline \multicolumn{2}{|c|}{$\begin{array}{l}\text { Numéro d'intervention } \\
\text { et de ligne }\end{array}$} & \multicolumn{2}{|c|}{ Claude } & \multicolumn{2}{|c|}{ Anémone } \\
\hline 15 & $1-4$ & I & & - expérience & \\
\hline 16 & $5-9$ & $\begin{array}{l}\text { - action (1) } \\
\text { - transfert (2 } \\
\text { - vécu (1) }\end{array}$ & & I & \\
\hline 17 & $\begin{array}{l}10+14 \\
+16-22\end{array}$ & 1 & & $\begin{array}{l}\text { - expérience } \\
\text { - vécu (2) } \\
\text { - transfert (1) }\end{array}$ & \\
\hline 18 & $24-25$ & - durée (1) & & I & \\
\hline 19 & $\begin{array}{l}26-28 \\
+30-31\end{array}$ & 1 & & $\begin{array}{l}\text { - vécu (2) } \\
\text { - transfert (2) } \\
\text { - expérience }\end{array}$ & \\
\hline Syn & hèse & $\begin{array}{l}\text { - durée } \\
\text { - action } \\
\text { - transfert } \\
\text { - vécu }\end{array}$ & $\begin{array}{l}=1 \\
=1 \\
=2 \\
=1\end{array}$ & $\begin{array}{l}\text { - expérience } \\
\text { - vécu } \\
\text { - transfert }\end{array}$ & $\begin{array}{l}=8 \\
=4 \\
=3\end{array}$ \\
\hline $\mathbf{T O}^{\prime}$ & $\mathbf{A L}$ & & $=5$ & & $=15$ \\
\hline
\end{tabular}

1 C'est-à-dire lorsqu'elles ne se contentent pas de la seule identification et de la seule mention de la présence ou de l'absence de telle ou telle* métaphore. 
Ces données chiffrées n'autorisent pas pour autant le recours à un arsenal de techniques quantitatives et à des traitements statistiques et cela pour une série de raisons qui doivent inciter à la vigilance. On peut ainsi être amené à travailler sur des petits nombres, pour lesquels un calcul de pourcentage frise le ridicule, si l'on prétend y avoir recours pour autre chose que de faciliter les comparaisons. Autre vigilance : les données quantitatives ne correspondront jamais qu'aux traces qui ont pu être relevées, sans que ce relevé puisse prétendre à l'exhaustivité. Ainsi, l'apparente certitude qui semble se dégager d'un nombre, recouvre en fait l'incertitude inhérente au caractère irréductiblement artisanal de l'étape de l'identification et du recensement des traces. Enfin, il ne s'agit pas de mélanger les genres en ayant recours ici à des techniques quantitatives, quand l'inspiration générale de la méthode est davantage du registre clinique.

Ces données consignées sous la forme de tableaux vont alors pouvoir servir de base à des analyses qui seront fonction des questions de recherche. Les commentaires des tableaux et les analyses ainsi fondées sont susceptibles de faire surgir de nouvelles questions particulières, des questions qui pourront amener le chercheur, soit à retourner au texte brut, soit à présenter les mêmes données d'une manière différente de celles qui paraissaient s'imposer, au vu des questions initiales. Nous retrouvons ici encore une certaine récursivité, déjà signalée ci-dessus quant à de nécessaires allers-retours entre l'identification des traces et la formulation des métaphores. A chacune des étapes, le chercheur est renvoyé à une interrogation sur ses pratiques, une interrogation abordée dans la dernière section de cet article.

\section{Exploration métaphorique et communication}

\subsection{Présentation de nouveaux extraits}

Que peut apporter cette besogneuse identification des métaphores ? En quoi peut-elle contribuer à l'approche des situations de communication? C'est ce qu'on va maintenant examiner synthétiquement dans cette troisième section. Pour cela, on aura recours à deux autres extraits des observations récoltées, ce qui permet d'une part d'illustrer autrement la méthode et d'autre part de la présenter ici en articulation avec des questions de recherches et des propositions théoriques. Après avoir présenté ces extraits ainsi que les traitements 
métaphoriques auxquels ils ont donné lieu, on pourra alors proposer une conception théorique de la communication qui rende compte de ces analyses.

\subsubsection{Présentation de l'extrait 2}

Dans une "mise en situation" trois étudiants sont invités à échanger sur les mesures à suggérer aux pouvoirs publics en matière de prévention de la toxicomanie. L'extrait choisi reprend les interventions des trois participants, lors du premier tour de table, au cours duquel chacun a eu l'occasion de s'exprimer une fois.

Animateur: (...) Qui souhaite commencer ? Monsieur Thomas X.

Thomas: (1) Ben, je peux, ben je vais peut-être eh, commencer par donner peut-être de mon point de vue, le point de olêpart qu'on peut prendre peut-être pour aborder un problème comme ça.

Animateur: Oui,

Thomas: (1) Et qui à mon avis, [il regarde l'animateur] consiste essentiellement dans une <connaissance approfondie du problème.> Donc, avmint d'intervernir par rapport st un problème, je crois qu'il serait vraiment urgent et important de bien le connaître. Connaître le problème, à la fois dans son ampleur, dans sa spécificité, dans ses méçmismes et dans, le resituer peut-être dans un ensemble. (...) Eh, pour pouvoir connaître ce problème, ben on est comfriontú à différentes difficultés, et notamment le fait qu'<il est toujours malaisé et difficile de connaître, de pouvoir évaluer quelque chose qui est interdit, [il regarde Dany] dans la mesure où c'est forcément caché, où l'accès à l'information est toujours difficile et en même temps, diabolisé, quoi.>(...) Donc, c'est peut-être une première piste que je donnerais, mais à mon avis, on, je pense qu'il y a une priorité qui devrait être mise d'abord dans les "o", je ne sais pas encore comment exactement, dans les organisations qui s'attacheraient à avoir une visionom, précise, nuancée et approfondlie du problème. Je pense que c'est en tout cas unge des premnières étapes.

Animateur: Madame, Valérie Z, peut-être ?

Valérie: (2) Oui, dans, dans le même ordre d'idée, quelque part, mais

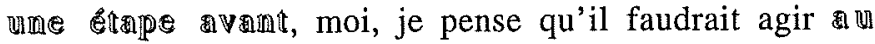
mìvegu de la prévention. Je pense qu'il y a beaucoup à faire, là. [elle regarde Thomas] Je pense qu'il y $\mathrm{a}$, une toxicomanie 
sociale qui est très importante, euh, des jeunes qui par besoin de reconnaissance, eh, vomi doms Iforicomaie, et je pense qu't ce mivegrolly, il faudrait faire quelque chose. C'est aussi très important. (...) Et je pense que ça peut remorer

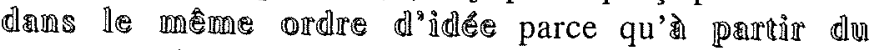
moment où on connaîtra eh, plus profomdenr, qui, comment, pourquoi, eh, ben, on saura qui (inaudible) avant, et comment.

Thomas : Non, ce n'est pas du tout incompatible, effectivement.

Animateur: Monsieur Dany Y.

Dany: (3) Ben, mon avis part ropport ça, c'est, ce serait de travailler principalement parr reipport à des,... des maisons de quartiers, etc. Parce qu'il me semble que jusqu'à présent $\mathbb{0}$ n ciblle un peu trop des choses, que ce soit mo miveande la toxicomanie ou d'autres malaises. La toxicomanie pour moi est un reflet d'un malaise tout à fait généralisé. Pour ma part, il est beaucoup moins important de comprendie ne profondeur le probleme, parce que je pense que c'est un peu utopique, et puis aussi du fait qu'il y a de l'urgence, face à ce genre de problème. Alors, voilà, ce serait ma première réaction.

\subsubsection{Commentaires relatifs à l'extrait 2}

Le travail d'identification des traces des logiques de raisonnement réalisé avec les juges permet notamment de dresser le tableau suivant. (Tab. X.2) et de procéder à quelques commentaires.

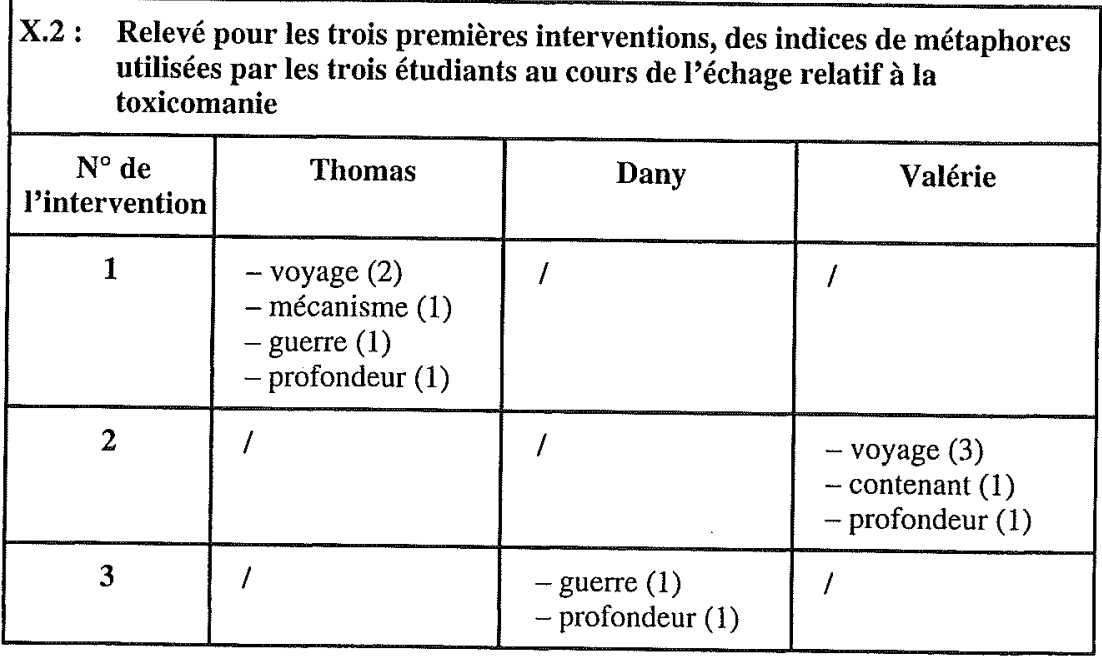


Dans la plupart de leurs interventions, les étudiants utilisent chacun plusieurs métaphores. Dans sa première intervention, le premier étudiant qui prend la parole (Thomas) a déjà recours à quatre des cinq métaphores qui seront utilisées par la suite. Si l'on fait référence cette fois à l'ensemble de l'échange, on notera qu'au terme des deux premières prises de parole, chacune des cinq métaphores repérées a déjà été utilisée au moins une fois. La cinquième métaphore, -celle du contenant- est utilisée dès la deuxième intervention de la séquence étudiée par Valérie. Et dès sa deuxième intervention personnelle, Thomas reprend à son compte cette cinquième métaphore, qui sera utilisée, quelques interventions plus tard, par le troisième protagoniste. Sur l'ensemble de l'échange, toutes ces métaphores sont utilisées par chaque participant, une, si pas plusieurs fois au cours de la discussion. Un tableau complet, portant sur l'ensemble de l'échange, permettrait de recomposer le trajet, la fortune ou l'infortune de chacune des métaphores identifiées, au fil de leurs reprises par les interlocuteurs.

\subsubsection{Présentation de l'extrait 3}

Après la projection d'un vidéogramme décrivant la situation écologique et sanitaire autour de la Mer d'Aral, consécutive à la culture intensive du coton dans la région, les étudiants sont invités à construire en sous-groupes un schéma qui identifie les boucles de rétroaction entre les éléments présentés dans le reportage. L'extrait choisi ici concerne un des sous-groupes, durant la réalisation concertée du schéma demandé.

Le travail réalisé avec les juges permet d'identifier dans les propositions des trois étudiants six logiques imagées de raisonnement ${ }^{1}$. Elles sont dégagées à partir de la production du sous-groupe, une production tenue à ce stade pour un seul discours. L'analyse de la dynamique de l'échange permet de constater l'existence de deux étapes. Dans un premier temps, les trois étudiants paraissent se

1 En l'occurrence, elles ont été formulées en ces termes :

- Expliquer, c'est chercher l'origine ;

- Expliquer, c'est rechercher et identifier une cause ;

- Expliquer, c'est constater l'existence ;

- Expliquer, c'est rendre compte d'une circulation ;

- Expliquer, c'est rendre compte des liens ;

- Expliquer, c'est rendre compte d'une transformation. 
contenter d'une énumération des différents éléments de la situation, davantage par volonté d'exhaustivité que par souci de relier entre eux ces éléments désordonnés. Un second temps correspond à la nécessité de produire un dessin commun, suite au rappel, par l'enseignante, du temps qui reste disponible. L'ensemble est consigné dans le tableau ci-dessous (Tab IX.2).

\begin{tabular}{|c|c|c|c|c|c|c|}
\hline & $\begin{array}{c}\text { 1ère } \\
\text { partie }\end{array}$ & $\begin{array}{c}\% \\
/ 99\end{array}$ & $\begin{array}{l}\text { 2ème } \\
\text { partie }\end{array}$ & $\begin{array}{c}\% \\
/ 119\end{array}$ & Total & $\begin{array}{c}\% \\
/ 218\end{array}$ \\
\hline Origine & 24 & 24 & 21 & 18 & 45 & 21 \\
\hline Cause & 27 & 27 & 23 & 19 & 50 & 23 \\
\hline Existence & 28 & 28 & 49 & 41 & 77 & 35 \\
\hline Transformation & 7 & 7 & 4 & 3 & 11 & 5 \\
\hline Circulation & 6 & 6 & 12 & 10 & 18 & 8 \\
\hline \multirow[t]{2}{*}{ Lien } & 7 & 7 & 10 & 8 & 17 & 8 \\
\hline & 99 & 100 & 119 & 100 & 218 & 100 \\
\hline
\end{tabular}

\subsubsection{Commentaires relatifs à l'extrait 3}

Quant aux productions spécifiques à chaque étudiant ${ }^{1}$, on peut notamment faire les observations suivantes :

* Si l'on se réfère à l'ensemble du texte, une observation s'impose : tous les étudiants ont recours à toutes les métaphores diagnostiquées, ce qui concorde avec l'observation déjà faite à propos de l'extrait 2 ;

* au Hit-Parade des métaphores, chaque étudiant ne les classe manifestement pas dans le même ordre, si l'on se fie à la fréquence des traces de l'usage de chacune d'elles. Si le trio de tête est constitué par l'Origine, la Cause et l'Existence, chaque étudiant ne privilégie pas les mêmes, tout au moins au cours de la première partie ;

1 Sur base d'un autre tableau détaillé, non présenté ici et qui restitue à chaque étudiant les traces de logiques imagées de raisonnement diagnostiquées dans leurs productions respectives. 
* par contre, sur l'ensemble de la seconde partie, leur nouveau classement respectif met chaque fois en tête la métaphore de l'Existence.

Conformément à la structure de cette séance de travail, la première partie compte peu d'interactions qui portent sur la négociation des modalités de la réalisation de leur tâche. Pour reprendre une distinction classique, les étudiants consacrent l'essentiel de leurs échanges à la production, tandis que le processus est au second plan. Dans la seconde partie, la proportion des échanges consacrés à la négociation du processus de fabrication du schéma demandé est nettement plus importante. En voici un extrait significatif.

Denise : $\quad$ C'est déjà clair comme ça, moi, je trouve.

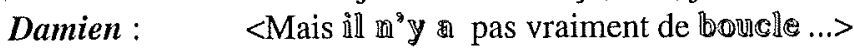

Ahmed : $\quad$ Allez, on avance, parce que nous n'avons plus de temps, et il est déjà clair... passons à l'autre schéma...

Damien : (qui prépare une nouvelle partie de grande feuille) $<$ Bon, comment va-t-on faire ?>

Denise : $\quad<$ Ce que tu pourrais déjà faire, des lìems, c'est des mpecheses de l'un à l'autre...>

Damien : On commence la mer d'Aral ?

Ahmed: $\quad$ Oui.

Denise : $\quad$ Mais allors, tu as les champs de coton. Et alors, çà change tout!

Damien : J'ai envie de lestres fleuves ici.

Denise : <Au-dessus de la mer? Ben oui, c'est lui qui alimente tout... $>$

Ahmed: $\quad$ Alors, ici...

Denise : $\quad<$ Moi, je mettrans "champs de coton" tout au dessus...>

Damien : $\quad$ Si on mell ça comme ça...

Ahmed : $\quad$ Fleuves dlispmirns ...

Denise : $\quad<$ Moi, j'aurais mìs "champs de coton" là (elle montre le haut de la feuille, sur la table)>

Damien : Les fleuves sont avdint les champs de coton.

Denise : Oui, mais champs de coton ìmplìque qu'ì] y æàt assèchement des fleuves et assèchement de la mer...

Une série d'interventions visent à s'accorder sur l'endroit où il convient de "mettre" les éléments relevés. Implicitement, la question de savoir par où on "commence" le dessin revient à négocier 
l'identification de l'endroit où les choses commencent, c'est-à-dire l'élément causal déterminant. Denise, en cohérence avec ses interventions précédentes, propose d'inscrire tout en haut de la page ce qui pour elle est la source-cause de tout ce qui est observé.

Denise : Mais álors, tu as les champs de coton. Et alors, ça change tout!

Denise : Mais je mettrais "champs de coton" tout au-dessus.

Pour l'étudiante, dans la mesure où les champs de coton représentent l'explication, la cause, la source de ce qui est constaté, c'est aussi au haut de la page qu'ils doivent figurer. Ce qui permet ensuite de "mettre" plus bas sur cette feuille les autres éléments relevés comme autant de conséquences qui découlent de cette source. Ce que vient confirmer cette nouvelle citation.

Denise : Moi, j' aurais mis "champs de coton" là... (elle montre le haut de la page)

Damien : Les fleuves sont avant les champs de coton

Denise : Oui, mais "champs de coton" implique qu'il y ait assèchement des fleuves...

Le fait pour un élément de se trouver sur la feuille au-dessus d'un autre signifie pour elle qu'il implique/cause cet autre élément.

Pour Damien, comme le montre son intervention dans le passage précédent, la préséance entre un élément et un autre signifie spécifiquement un rapport chronologique d'antériorité. Le terme "avant" qu'il utilise associe la dimension temporelle, quant aux processus dont il s'agit de rendre compte et la dimension spatiale, quant à la nécessité d'une représentation graphique. On constate alors que s'il partage avec Denise l' orientation Haut-Bas de la feuille, ce n'est pas à la relation causale qu'elle est associée mais au rapport chronologique entre les éléments qu'il y a lieu de "mettre" dans le schéma.

En ce qui concerne Ahmed maintenant, force est de constater que dans le deuxième extrait, ses interventions ne permettent pas d'identifier la façon dont il conçoit les rapports entre les différents éléments de la situation et leur place dans le schéma.

Cette analyse permet alors de contraster deux conceptions du rapport entre les faits à comprendre et leur représentation schématique graphique. 


\begin{tabular}{|l|l|l|}
\hline & Représentation graphique & Phénomènes à comprendre \\
\hline DAMIEN & Haut & Avant \\
& Bas & Après \\
\hline \multirow{2}{*}{ DENISE } & Haut & Cause(s) \\
& Bas & Conséquence $(\mathrm{s})^{1}$ \\
\hline
\end{tabular}

Sur base de cette schématisation de l'analyse qui vient d'être faite, quelles considérations plus générales peut-on tenter de tirer à ce
stade?

La lecture faite plus haut de l'ensemble du travail de ce sousgroupe constatait l'existence de deux temps. La première partie semblait consister en la juxtaposition, tant de divers faits que de leurs conceptions respectives. La seconde partie, pressée par le rappel de l'échéance et la nécessité de produire un schéma commun, contraignait les étudiants à une négociation entre leurs conceptions respectives. Cette obligation de négocier fait en sorte que le dessin représente une médiation par laquelle leurs divergences doivent
trouver à s'articuler.

On aurait pu croire que la nécessité de négocier allait amener les participants à modifier les métaphores qu'ils utilisent pour appréhender la situation. Or, on voit certes apparaître un accord portant sur l'orientation Haut/Bas de la page et la disposition des différents éléments de la situation à comprendre, le long de cet axe. Mais cet

\footnotetext{
1 L'association de l'orientation Haut-Bas avec le couple Causes-Conséquences ou raisonnement causal avec Source-<embouchure>autoriserait l'association de fois au "début" du travail de ce sousulpabilité. Le terme de "paradis" est cité trois "décision administrative" qui débouchroupe (paradis agricole) tandis que c'est une qui aurait précipité "la chute". Denise sur une série de conséquences négatives et On peut imaginer que ça a été vraiment pose d'ailleurs explicitement la question : " quasi définitoire de notre culture, on peut au..." Outre la question de la culpabilité, métaphore dégagée par Lakoff : "LE-Bssi souligner la correspondance avec la MALHEUR-EST-EN-BAS". Suggéré ici au simple titre d'ébau-HAUT" + "LEpoint mériterait sans doute d'autres développements que d'ébauche d'hypothèse, ce cette note infrapaginale. Une autre fois, peut-être...
} 
accord de surface polarise à un autre niveau des différences entre deux des protagonistes. Leur accord apparent sur cette orientation Haut/Bas dissimule en fait un désaccord quant à leurs associations respectives aux deux pôles de cette orientation. Au terme de cet "échange", c'est la conception de Denise qui l'emporte, tandis que Damien, après quelques protestations irritées sur le manque de temps, semblera renoncer à faire valoir sa perception des choses.

\subsection{Constats généraux}

L'exploration métaphorique que cet extrait permet d'illustrer davantage, a été guidée ici par la question de recherche suivante : "Comment rendre compte des modalités d'interaction lors d'échanges au cours desquels les interlocuteurs ont recours à des métaphores différentes ?"

La réponse à cette question est nette: tous les utilisent toutes! Tous les participants à un échange utilisent toutes les métaphores repérées sur base des productions verbales manifestées dans les échanges métaphoriquement analysés. Ainsi, cette diversité de métaphores n'apparaît pas comme un obstacle à la communication, entendue ici comme inter-compréhension, mais au contraire comme (un des) moyen(s) par le(s)quel(s) chacun peut comprendre l'autre et percevoir qu'il est compris de lui. Si des personnes engagées dans une interaction se comprennent, tout en ayant recours à des métaphores différentes, il faut conclure que cette diversité métaphorique ne se présente pas, communicationnellement parlant, comme un obstacle à leur intercompréhension. Cette diversité même qui permet le recours aux métaphores utilisées par les autres est un procédé par lequel se construit cette intercompréhension. Pour le dire autrement. Si des personnes en situation de communication se comprennent, tout en mobilisant des métaphores différentes, c'est non pas malgré le fait qu'elles ont recours à des métaphores différentes, mais tout au contraire parce que chacune a recours à toutes les métaphores utilisées par les autres!

Plus, l'obligation qui est celle des étudiants de produire une analyse commune d'une situation complexe les amène à se concerter, non seulement sur ce qu'on appelle communément la compréhension de la situation en question ou encore sur les modalités de réalisation de la tâche, mais aussi sur les métaphores à partir desquelles ils vont 
ensemble la concevoir ${ }^{1}$. Si une même situation est susceptible d'être appréhendée à partir de plusieurs métaphores, il reste que toutes les métaphores ne sont pas pertinentes pour en rendre compte. L'appréciation du degré de pertinence de chacune des métaphores évoquées dans la discussion générale est très précisément ce qui va faire l'objet d'une négociation implicite.

\subsection{Communiquer : négocier des métaphores}

Sur un registre théorique, ces observations et considérations permettent de proposer une lecture des situations de communication en termes de négociation de métaphores. En articulant ces considérations et les propositions de Lakoff, une situation de communication peut être conçue dans les termes suivants.

Du point de chaque participant à une situation de communication, participer à un échange ${ }^{2}$ revient -notamment- à proposer des structures schématiques et imagées comme autant de façons de concevoir ce qui fait l'objet ou les objets de l'échange. Chacun est amené à sélectionner, dans un ensemble de métaphores potentiellement disponibles en fonction de son bagage culturel, les métaphores qu'il estime pertinentes pour appréhender le thème de la discussion. La contribution de chaque partenaire tient en sa capacité de proposer des métaphores et à les mettre ainsi plus particulièrement à disposition des autres. Ce qui revient aussi à en sélectionner l'une ou l'autre, potentiellement pertinentes, parmi l'ensemble de celles que lui offre sa/leur culture. Ces références culturelles constituent ainsi un "stock" à la commune disposition de chacun d'eux. Chaque métaphore

1 Précisément parce que cette compréhension implique nécessairement le recours à des structures schématiques et imagés, à des logiques imagées de raisonnement.

2 On retrouve dans cette expression la référence à une affirmation chère à l'approche pragmatique psycho-sociologique, que formule Ray Birdwhistell : "Un individu ne communique pas ; il prend part à une communication (...) il n'est pas l'auteur de la communication, il y participe. La communication, en tant que système, ne doit donc pas être conçue sur le modèle élémentaire de l'action et de la réaction, si complexe soit l'énoncé. En tant que système, on doit la saisir au niveau de l'échange" (R. L. BIRDWHISTELL, "Contribution of Linguistic-Kinetic studies to the understanding of schizophrenia", in A. AUERCBACH, Schizophrenia - An integrated approach, New York, The Ronald Press Company, 1959, pp. 99-123. Page 104). Cité par P. WATZLAWICK, J. H. BeAvin, D. D. JACKSON, Une Logique de la Communication. (Traduit de l'américain ; 1967 pour l'édition originale), Paris, Éd. du Seuil, coll. "Points", 1972, p. 68. 
proposée se présente pour les autres participants comme une structure schématique et imagée qu'eux-mêmes auraient tout aussi bien pu puiser et proposer comme leur contribution métaphorique à l'échange. Avoir recours à une métaphore déterminée revient à la mettre en évidence, parmi un ensemble de métaphores virtuellement pertinentes.

Toujours du point de vue de chaque participant, prendre part à une communication consiste également à reprendre on ne pas reprendre les différentes propositions de métaphores qui sont faites par les autres interlocuteurs, tout comme les autres font de même.

Conçue maintenant au niveau de l'interaction et de ses règles -et non plus au niveau des participations individuelles-la communication peut être appréhendée comme une négociation coopérative dont l'enjeu est l'édification d'une constellation de métaphores signifiantes. Cette négociation se déroule selon des modalités suivantes. Si les parties prenantes à une situation de communication peuvent donc chacune proposer une métaphore et reprendre à leur compte les métaphores proposées par les autres, il leur est tout autant possible de ne pas reprendre l'une ou l'autre des métaphores qui sont ainsi mises à la disposition des interlocuteurs. Chaque intervenant procède de la sorte. Dès lors, au fil de l'échange, certaines métaphores seront davantage utilisées, tandis que d'autres ne le seront que peu, voire pas du tout. Parmi l'ensemble des structures schématiques et imagées potentiellement pertinentes par rapport à l'objet de l'échange, seule une partie peut ainsi être effectivement utilisée, par le jeu de propositions et des reprises. L'ensemble signifiant que constitueront in fine les métaphores sélectionnées au cours du processus ne dépendra donc que pour une part des activités cognitives individuelles. Cet ensemble dépendra également des ressources métaphoriques de la culture (ou la sous-culture) concernée et dans une large mesure des modalités de l'interaction. Cet ensemble signifiant sera donc davantage une construction collective, négociée et coopérée, une émergence de la configuration particulière qu'auront prises les modalités de cet échange. Il sera le fait de la communauté interprétative que constitue les interlocuteurs, une communauté qui se construit ainsi comme telle en même temps qu'elle élabore cette signification située.

A un plan épistémologique, cette conception se veut aussi constructiviste, en ce que le sens attribué à un objet au cours de l'échange dépendra moins de ce que cet objet peut être en soi, mais davantage de l'ensemble singulier de métaphores qui émergera de la communication, selon les modalités que nous avons présentées. 
Le caractère relativement singulier, temporaire, éphémère de cet univers de sens qui ne serait donc valable que pour le temps de l'interaction, pose évidemment la question de savoir ce que deviennent cet univers et ces métaphores au terme de l'interaction. Le fait pour une métaphore d'avoir été retenue comme élément d'un univers de sens réunissant des partenaires au sein d'une situation de communication déterminée augmente la probabilité qu'elle soit à nouveau convoquée par ces ex-partenaires dans des situations de communication ultérieures, avec ces mêmes partenaires ou avec d'autres. Confrontée à d'autres dans de nouvelles interactions, cette métaphore sera à nouveau au centre de l'enjeu de ces autres constructions de sens et peut ainsi se trouver mise à la disposition d'autres partenaires. A leur tour, ils y auront recours dans d'autres contextes interactionnels.

$\mathrm{Au}$ gré de ses participations à de multiples interactions, chaque interlocuteur contribue ainsi à l'augmentation ou à la restriction d'une métaphore donnée. On pourrait ainsi assister, pour peu que l'on dispose des outils adéquats, à la diffusion des métaphores qui ont cours dans une culture ou une sous-culture, un groupe professionnel ou une classe sociale, une classe d'âge ou une époque...

\section{La méthode : considérations générales}

$\mathrm{Au}$ moment de conclure, on relèvera quelques éléments de réflexion de portée plus générale, relatifs à cette méthode d'exploration métaphorique des productions langagières, en commençant par un des aspects de sa mise en œuvre : son caractère réitératif.

\subsection{Une certaine récursivité}

Le trajet linéaire orthodoxe, censé partir des questions de recherche, passer par la collecte, l'organisation puis le traitement des données, pour se terminer par l'analyse de cet ensemble est ici quelque peu perturbé. Il se boucle sur lui-même en l'un ou l'autre endroit. Les réflexions et les élaborations formulées à une étape sont susceptibles d'influer, non seulement sur les étapes qui logiquement la suivent mais aussi sur celles qui, toujours aussi logiquement, la précèdent. Par exemple, les premières analyses menées sur base d'une certaine présentation des données feront vraisemblablement apparaitre de nouvelles supputations qui, pour les éprouver, vont nécessiter le 
recours à une autre présentation des données, une présentation qui à son tour, pourrait exiger le retour au matériau de base.

On peut donc ainsi être amené à modifier, déplacer, perfectionner, enrichir l'analyse par l'explicitation et l'exploration de nouvelles questions particulières, dont la formulation était inenvisageable $a$ priori. Elles émergent au contraire progressivement de la rencontre entre d'une part les préoccupations du chercheur, d'autre part la méthode dans sa mise en oeuvre et de troisième part des matériaux dans leurs caractéristiques formelles et de contenus. Si les matériaux doivent être manipulés de manière à les rendre appréhendables par la méthode, cette dernière doit tout autant être adaptée aux traits essentiels et pertinents des matériaux. Ceux-ci doivent se plier aux exigences de la méthode qui elle-même doit s'ajuster aux matériaux et aux questions de recherche. Forçons le trait: dans une certaine mesure, c'est donc aussi le matériau lui-même qui oriente la formulation des questions que l'on se posera à son endroit et les portions de la méthode avec lesquelles on va aborder l'ensemble.

Insistons sur ce point : il ne s'agit pas de tenir le caractère réitératif que nous relevons ici comme une faiblesse, voire une tare de la méthode. Il s'agit tout au contraire d'y voir une des conditions de déploiement de ses vertus heuristiques.

\subsection{Les limites de la formalisation}

La volonté d'explicitation par le menu les détails des différents opérations atteint çà et là des limites. A chacune de étapes, il y a lieu de tabler sur l'expérience, la vigilance, le savoir-faire et la perspicacité du chercheur. Par exemple, il aura à s'appuyer tout à la fois sur ce qu'il sait par ailleurs des personnes dont les discours sont étudiés, sur ses "impressions" lors de la collecte des matériaux, sur sa connaissance du contexte... tout comme il aura à se méfier de cet ensemble. Et nous pourrions ajouter, à la suite d'Edgar Morin :

(...) il nous faut nous méfier de notre confiance, mais aussi nous méfier de notre méfiance ${ }^{1}$.

1 E. MorIn, Pour sortir du XX $X^{e}$ siècle, Paris, Fernand Nathan, 1981 (Rééd. Éd. du Seuil, coll. "Points") p. 25. 
C'est dans cette perspective qu'il s'agit de considérer le recours à des juges : examinons ce point.

Ce que permet le recours à des juges, c'est l'obligation pour le chercheur d'expliciter et de justifier chacune des opérations qu'il mène, des choix qu'il pose, des options qu'il prend. Ce que permet le recours à des juges, c'est la nécessité de rendre compte de ses propres raisonnements, de les rendre intelligibles et donc critiquables. Ce qui est finalement une exigence de toute production scientifique. Ce que permet de recours à des juges, c'est précisément le fait qu'il s'agit, non de découvrir une vérité quelconque -dissimulée sous les mots, en l'occurrence- mais qu'il s'agit d'inférer, de construire, bref de juger et que ce jugement est irréductiblement une production intellectuelle située. L'exigence scientifique consiste, non à adopter "le point de vue de nulle part", mais tout au contraire à donner à voir le point de vue adopté et de faire de ce point de vue un objet de réflexion significatif. En l'occurrence, on parlera donc d'intersubjectivité et de construction plutôt que d'objectivité et de vérité à "découvrir"...

L'appel à des juges rappelle et renforce cette exigence, comme le signale le même Edgar Morin :

Il est une révolution copernicienne, inhérente à la réforme des structures de pensée, et que chacun peut effectuer : c'est d'inclure dans toute observation l'auto-observation, dans tout examen l'auto-examen, d'introduire dans toute connaissance la volonté d'auto-connaissance du connaissant. (...) La logique de l'auto-examen empêche l'auto-examen de se refermer sur luimême : en effet, elle appelle l'examen de l'auto-examen, c'està-dire le recours au dialogue et la discussion avec des examinateurs extérieurs ${ }^{1}$.

Aussi loin que l'on veuille aller dans la direction d'une description méticuleuse des étapes d'une procédure d'identification des métaphores, il restera toujours une limite, qu'il y a lieu de concevoir, non comme une lacune qui ravalerait la méthode au rang des seconds choix mais comme un rappel d'une exigence propre à toute démarche scientifique : celle de la réflexion.

1 Ibid., pp. 166-167. 


\subsection{Une relation observateur-sujet}

Une autre préoccupation mérite d'être sinon approfondie, du moins brièvement discutée: celle du rapport entre le chercheur et les sujets dont les discours sont ainsi explorés.

Dans une perspective interactionnelle, cette relation observateursujet(s) est à appréhender comme une relation de pouvoir, mais une relation ambiguë. A un niveau, la tentation du chercheur est d'adopter une position haute, c'est-à-dire une position depuis laquelle il entend pouvoir scruter le plus intime des pensées des sujets. Ce faisant, il attribue aux sujets une position basse, c'est-à-dire une position depuis laquelle ces sujets sont censés en savoir moins que lui sur leurs propres pensées. A un autre niveau, le chercheur dépend de la (bonne) volonté de ces sujets. Il a besoin de leur collaboration dans la mesure où ce sont eux qui lui fournissent (ou non) les matériaux de base pour la recherche qu'il mène. Dans de telles configurations relationnelles, on risque de voir se mettre en place, soit des jeux de séduction, mutuelle ou non, entre le chercheur qui souhaite avoir l'accord des sujets et ces sujets tentés de "négocier" cet accord en échange de l'un ou l'autre des avantages que pourrait leur offrir le contexte. On pourrait aisément comparer cette situation à celle d'un photographe qui, cherchant à faire des portraits de reportage, est confronté au dilemme classique, dont les extrêmes pourraient être formulés ainsi. Soit, il prend des photos à la sauvette, tel un voleur-voyeur, s'exposant alors à l'une ou l'autre "représailles", s'il se fait repérer. Soit, il prend contact avec un sujet et lui achète le droit de le "prendre" en photo, avec le risque d'être accusé de collusion et de malhonnêteté. Au-delà de solutions pratiques ou des attitudes générales qui peuvent être prônées, -dans le cas présent, la contractualisation- il importe que le chercheur fasse aussi de ce point un objet de réflexion et de vigilance, non seulement du point de vue du bénéfice pour sa recherche, mais aussi dans sa dimension éthique. "Qui suis-je pour observer ainsi, pour poser de telles questions, pour mettre l'autre dans une telle situation, qui suis-je pour savoir autant, autrement, mieux que lui ce qu'il pense, voire apporter des réponses à sa place..." Ne pas affronter cette interrogation reviendrait à considérer qu'il est légitime d'instrumentaliser non seulement son discours, mais le sujet lui-même, ce qui est une position éthiquement bien peu défendable. 


\subsection{Réflexion permanente}

Terminons ces considérations générales par une affirmation banale aussitôt qu'elle est formulée, pourtant cruciale, aussitôt qu'elle est perdue de vue : l'obligation de réfléchir.

Soulignant le caractère réitératif de la méthode et l'indépassable limite à laquelle se heurte toute tentative de formalisation, on a insisté sur ce point: cette méthode ne s'avère intéressante que dans la mesure où elle fait se rencontrer des questions de recherche, des matériaux concrets et un chercheur vigilant. Dans la mesure où la méthode présentée ici se range dans la catégorie de celles qui nécessitent des allers-retours fréquents entre les questions que l'on se pose, les hypothèses que l'on propose et les matériaux sur lesquels on travaille, la mise en oeuvre de cette méthode exige une clairvoyance incessante, non seulement quant à la méticulosité dans l'usage de tel ou tel outil, mais aussi quant à la cohérence de l'ensemble. Il doit ainsi éviter tout à la fois de forcer le matériau au-delà de ce qu'il peut apporter, inversement de suivre la richesse et la logique du matériau au point d'en oublier ses propres questions initiales ; il doit éviter de se figer exagérément sur ces questions, à peine de négliger la richesse du matériau. Il devra enfin s'efforcer de choisir les outils en cohérence, tant avec les questions posées que les caractéristiques formelles et les contenus des matériaux à explorer.

Ces considérations permettent de souligner, en guide de conclusion, la dimension artisanale de cet ensemble qui, quoiqu'il comporte un certain nombre de techniques rigoureuses, n'en demeure pas moins dépendant des qualités du chercheur. Si ce vocable d'artisan peut présenter, en l'un ou l'autre contexte, une résonnance péjorative, il n'en va pas ainsi dans notre chef. Le terme évoquerait davantage, outre le caractère besogneux, l'unicité de la pièce finale qui condense ainsi, en un seul et même objet, la qualité des matériaux, la pertinence dans le choix des outils et le savoir-faire de l'artisan. 